\title{
Ruptured Ectopic Pregnancy in Non-Commuicating Rudimentary Horn of Bicornuate Uterus- A Case report and Review of literature
}

\author{
Authors \\ Varsha Shrivastava', Vaibhav Vikas ${ }^{2}$ \\ ${ }^{1}$ Gandhi Medical College, Bhopal, Madhya Pradesh, India \\ ${ }^{2}$ Government Medical College, Thiruvananthapuram, Kerala, India
}

\begin{abstract}
\section{Introduction}

The incidence of mullerian duct anomalies in general population is found to be $3.2 \%$. Unicornuate uterus occurs in 1 in 4020 women in the general population and a rudimentary horn is present in about $84 \%$ of the cases ${ }^{[1]}$. The incidence of pregnancy in the rudimentary horn is approx $1: 100000$ to $1: 140000^{[2]}$. The first case was reported by Maricean in $169^{[3],[4]}$. Pregnancy in the rudimentary horn usually ends in rupture during second trimester in about $90 \%$ of the cases.
\end{abstract}

Pregnancy in a rudimentary horn of bicornuate uterus is an extremely rare form of ectopic gestation which occurs most commonly in a noncommunicating cavitary horn and ruptured form is even more rarer. The rudimentary horn may (as in majority of cases) or may not communicate with the uterine cavity. We report one such rare case of ruptured pregnancy of non communicating rudimentary horn of bicornuate uterus in a young multiparous female. Diagnosis was made after laparotomy was performed for ruptured ectopic pregnancy. Excision of the rudimentary horn with ipsilateral salpingectomy was done.

Keywords- Bicornuate/Unicornuate uterus, Rudimentary Horn, Communicating/noncommunicating

\section{Case Report}

A 24 years old female presented to the emergency department of Obstetrics and Gynaecology with complains of amenorrhoea since 4 months (L.M.P. 30th October 2013) and severe pain in whole abdomen since 8 hours. Patient was G3P2L1A1 (gravida 3, 1 live birth, 1 abortion). On clinical examination, patient appeared severely pale, had pulse of 120/min, blood pressure $90 / 70 \mathrm{~mm}$ of $\mathrm{Hg}$, a mass was palpable just above the pubic symphysis, firm in consistency, irregularly defined margins, tender to touch. Abdomen was grossly distended with moderate tenderness, no guarding, and evidence of free intraperitoneal fluid present. Urine pregnancy test was positive. Culdocentesis through cul-de-sac showed non clotted blood. Provisional diagnosis of Acute abdomen with haemoperitoneum with possible ruptured ectopic pregnancy was made. Ultrasonography of abdomen and pelvis revealed ruptured live ectopic pregnancy of approximately 13 weeks 5 days with moderate free fluid in pelvic and peritoneal cavity. Urgent exploratory laparotomy (figure 1,2,3) was performed which revealed (1) Gross hemoperitoneum of $1500 \mathrm{ml}$; (2) Uterus was bicornuate with left horn being rudimentary; (3) Rudimentary horn was ruptured with the gestational sac of about $10 \mathrm{cms}$ X $6 \mathrm{cms}$ lying in the peritoneal 
cavity; (4) Placenta of the gestation sac was attached at ruptured site on the uterus; (5) Rudimentary horn was not communicating with the normal uterine cavity. (6) Left fallopian tube was attached to the rudimentary horn. Excision of rudimentary horn with ipsilateral salpingectomy was done. Post operative course was uneventful and patient was discharged on eighth day.
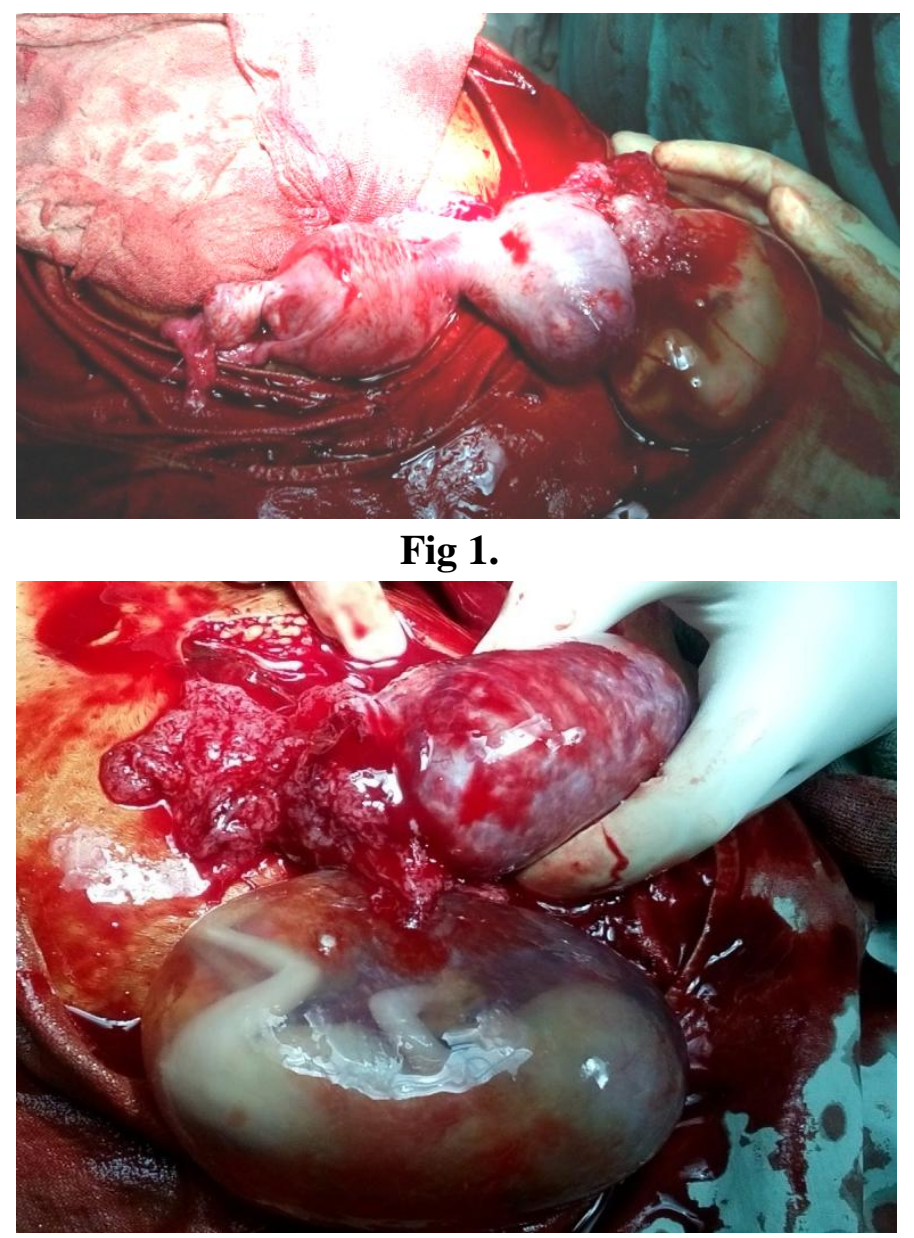

Fig 2.

Fig 1 \& 2. - Intraoperative view of gestational sac with foetus coming out from ruptured left rudimentary horn of uterus.

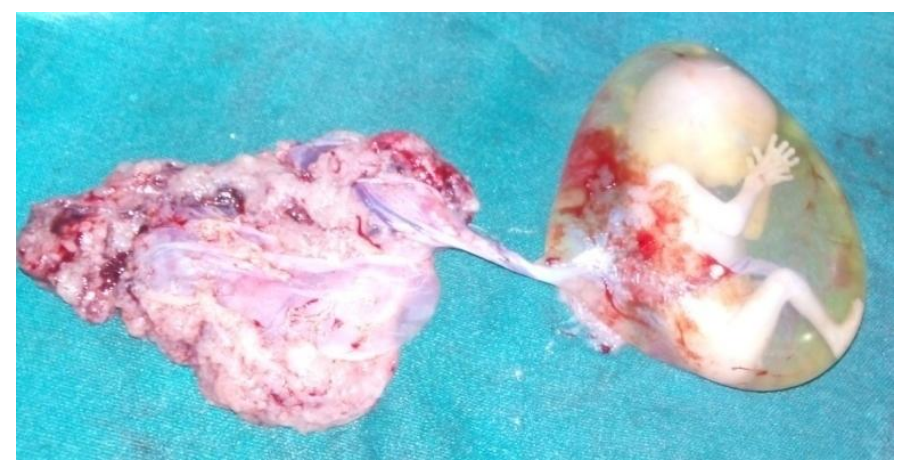

Fig 3. Removed Gestational sac with its placenta.

\section{Discussion}

Unicornuate uterus results due to partial or complete failure of development of one of the paramesonephric ducts. Partial development gives rise to a rudimentary uterine horn which can be further subclassified in to communicating, noncommunicating, no cavity, and no horn ${ }^{[1]}$.

Pregnancy in a non-communicating rudimentary horn occurs through the transperitoneal migration of the spermatozoon or the fertilized ovum, as evidenced by the $10 \%$ prevalence of a corpus luteum on the contralateral side ${ }^{[2]}$. The timing of rupture of such pregnancy varies from case to case because of the varied muscular constitution in the thickness and distensibility of the wall of the rudimentary horn. Rupture of such pregnancy often occur in second or third trimester which in most cases results in life threatening hemorrhage leading to shock ${ }^{[5]-[9]}$. Unlike tubal ectopic pregnancy, bleeding is more severe in rupture of the rudimentary horn as the uterine wall is much thicker and more vascular. Few cases of pregnancies with late or false diagnosis were progressed to third trimester resulting in live births have been reported $^{[9]}$.

Early diagnosis of the condition can be challenging but very essential as its associated with significant mortality. Ultrasound, hysterosalpingogram, hysteronscopy, laparoscopy, and MRI are the diagnostic tools normally used ${ }^{[10]}$. Tsafrir et al ${ }^{[4]}$ has proposed ultrasonographic criterias for diagnosing pregnancy in the rudimentary horn: (1) a pseudo pattern of asymmetrical bicornuate uterus; (2) absent visual continuity tissue surrounding the gestation sac and the uterine cervix; (3) presence of myometrial tissue surrounding the gestational sac. Sensitivity of ultrasound is only $26 \%$ which further decreases as the pregnancy advances ${ }^{[11]}$. It can be very easily misjudged as tubal pregnancy, cornual pregnancy, intrauterine pregnancy, and abdominal pregnancy in experienced hands ${ }^{[12]}$. Magnetic resonance imaging (MRI) is very useful not only in confirming the diagnosis but also for planning the surgery ${ }^{[13]}$. Nonetheless most cases remain undiagnosed until it ruptures and presents as an emergency. 
The established primary strategy of management of rudimentary horn is surgical removal. There has been incidences of misdiagnoses where labour induction agents were used for termination of pregnancy in a rudimentary horn leading to rupture of the horn ${ }^{[14]}$. Immediate surgery is recommended by most after the diagnosis even in unruptured cases [8],[11]. Prophylactic removal of the horn prior to pregnancy to prevent complications is also practised. Medical management with methotrexate and its resection by laparoscopy (advocated in the recent decade) is also reported. Renal anomalies are found in $36 \%$ of cases ${ }^{[11]}$ which makes further assessment of these women mandatory.

\section{Conclusion}

Non communicating rudimentary horn pregnancy is an entity associated with life threatening consequences; however early diagnosis and intervention avoids maternal morbidity and mortality significantly. A high index of suspicion is warranted to detect this rare variation of pregnancy before uterine rupture occurs. Despite advances in ultrasound and other diagnostic modalities, laparotomy holds the place of confirmatory diagnostic modality. Timely resuscitation, surgery, and blood transfusion are needed to save the patient. Excision of rudimentary horn is advised whenever detected in the reproductive period of life.

\section{References}

1. Reichman D, Laufer MR, Robinson BK. Pregnancy outcomes in unicornuate uteri: a review. Fertil Steril 2009;91:1886-94.

2. O'Leary JL, O'Leary JA. Rudimentary horn pregnancy. Obstet Gynecol 1963;22:371-5.

3. Elsayegh A, Nwosu EC. Rupture of pregnancy in the communicating rudimentary uterine horn at 34 weeks.Human Reprod. 1998; 13 (12) : 3566-68. 52.

4. Tsafrir A, Rojansky N, Sela HY, GomoriJm, Nadjari M. Rudimentry horn pregnancy. First trimester prerupture sonographic diagnosis and confirmation by magnetic resonance imaging.J Ultrasound Med 2005;24:219-23.
5. Jain R,Gami N,Puri M, Trivedi SS.A rare case of intact rudimentary horn pregnancy presenting as haemoperitoneum.J Hum Reprod Sci 2010;3(2):113- 115.

6. Henriet E, Roman H, Zanati J,Lebreton B, Sabourin JC Loic M.Pregnant noncommunicating rudimentary uterine horn with placenta percreta. Journal of the Society of Laproendoscopic Surgeons 2008;12:101-103.

7. Mauriceau F. Traite des maladies des femmes Grosses vol. 1, Compaigne des libraries, Paris, France, 1721.4.

8. Nahum GG. Rudimentary uterine horn pregnancy. The 20th-century worldwide experience of 588 cases. J Reprod Med 2002;47:151-63.

9. Shin JW, Kim HJ. Case of live birth in a non-communicating rudimentary horn pregnancy. J Obstet Gynaecol Res 2005;31:329-31.

10. B. P. Lawhon, J. R.Wax, and R. T. Dufort, "Rudimentary uterine horn pregnancy diagnosed with magnetic resonance imaging," Obstetrics and Gynecology, vol. 91, no. 5, p. 869, 1998.

11. Jayasinghe Y, Rane A, Stalewski H, Grover $\mathrm{S}$. The presentation and early diagnosis of the rudimentary uterine horn. Obstet Gynecol 2005;105:1456-67.

12. F. Bahadori, S. Borna, T. Behroozlak, S. Hoseini, and H. Ayatollahi, "Failed induction in second trimester due to pregnancy in an uncommunicated rudimentary horn: case report," Journal of Family and Reproductive Health, vol. 3, no. 3, pp. 95-97, 2009.

13. Lawhon BP, Wax JR, Dufort RT. Rudimentary uterine horn pregnancy diagnosed with magnetic resonance imaging. Obstet Gynecol 1998;91 (5 Pt 2):869.

14. T. A. Samuels and A. Awonuga, "Secondtrimester rudimentary uterine horn pregnancy: rupture after labor induction with misoprostol," Obstetrics and Gynecology, vol. 106, no. 5, pp. 1160-1162, 2005. 